\title{
The Role of Substance $P$ in Pulmonary Clearance of Bacteria in Comparative Injury Models
}

Terry Hsieh, ${ }^{*}$ Max H. Vaickus, ${ }^{*}$ Thor D. Stein, ${ }^{* \dagger}$ Bethany L. Lussier, ${ }^{*}$ Jiyoun Kim, * David M. Stepien, ${ }^{*}$ Elizabeth R. Duffy, * Evan L. Chiswick, ${ }^{*}$ and Daniel G. Remick*

From the Department of Pathology and Laboratory Medicine, ${ }^{*}$ Boston University School of Medicine, Boston; the VA Boston Healthcare System, ${ }^{\dagger}$ Boston; and the Department of Veterans Affairs Medical Center,${ }^{\ddagger}$ Bedford, Massachusetts

Accepted for publication August 29, 2016.

Address correspondence to Daniel G. Remick, M.D., Department of Pathology and Laboratory Medicine, Boston University School of Medicine, 670 Albany St., 4th Floor, Boston, MA 02118. E-mail: remickd@bu.edu.

\begin{abstract}
Neural input to the immune system can alter its ability to clear pathogens effectively. Patients suffering mild traumatic brain injury (mTBI) have shown reduced rates of pneumonia and a murine model replicated these findings, with better overall survival of TBI mice compared with sham-injured mice. To further investigate the mechanism of improved host response in TBI mice, this study developed and characterized a mild tail trauma model of similar severity to mild TBI. Both mild tail trauma and TBI induced similar systemic changes that normalized within 48 hours, including release of substance $P$. Examination of tissues showed that injuries are limited to the target tissue (ie, tail in tail trauma, brain in $\mathrm{mTBI}$ ). Pneumonia challenge showed that mild TBI mice showed improved immune responses, characterized by the following: i) increased survival, ii) increased pulmonary neutrophil recruitment, iii) increased bacterial clearance, and iv) increased phagocytic cell killing of bacteria compared with tail trauma. Administration of a neurokinin-1-receptor antagonist to block substance $\mathrm{P}$ signaling eliminated the improved survival of mTBI mice. Neurokinin-1-receptor antagonism did not alter pneumonia mortality in tail trauma mice. These data show that immune benefits of trauma are specific to mTBI and that tail trauma is an appropriate control for future studies aimed at elucidating the mechanisms of improved innate immune responses in mTBI mice. (Am J Pathol 2016, 186: 3236-3245; http:// dx.doi.org/10.1016/j.ajpath.2016.08.014)
\end{abstract}

Neural input exerts significant control on the ability of the immune system to clear pathogens effectively. Early studies have shown that stress has a profound detrimental effect on the immune response. ${ }^{1}$ Psychologic or physiologic stress can result in dysregulation of these pathways, such as chronic activation, which ultimately leads to immunosuppression. ${ }^{2}$ Activation of the vagus nerve also has been shown to induce powerful anti-inflammatory effects through the $\alpha 7$ nicotinic acetylcholine receptor. In severe traumatic brain injury (TBI) patients, it has been proposed that hyperactivity of the vagus depresses immune responses through strong dampening of proinflammatory mediator production. ${ }^{3}$ In contrast to these studies showing that the neuroimmune axis decreases immune responses, our previous work documented that head trauma patients showed significantly reduced rates of pneumonia compared with blunt trauma patients. ${ }^{4} \mathrm{~A}$ murine model of mild traumatic brain injury (mTBI) was able to reproduce these findings with enhanced resistance to bacterial pneumonia compared with sham injury mice. ${ }^{4}$ mTBI mice showed improved survival, augmented pulmonary neutrophil recruitment, and reduced bacterial burdens compared with sham-injured mice. These findings show that neuroimmune modulation can show beneficial effects by improving immune function. Further investigation into the mechanisms by which mTBI augments the innate immune response could offer valuable insight into fighting infections in today's increasingly

Supported by grants R01 GM82962, R21 AI112887, R01 GM97320, T32 HL 007501, T32 GM 86308, T32 HL 007035, and T32 AI 007309.

Disclosures: None declared.

Current address of D.M.S., Section of Plastic Surgery, Department of Surgery, University of Michigan Medical School, Ann Arbor, MI; of E.R.D., Boston Children's Hospital, Harvard Medical School, Boston, MA; of E.L.C., Department of Biological Engineering, Massachusetts Institute of Technology, Cambridge, MA. 
antibiotic-resistant world (CDC, http://www.cdc.gov/ drugresistance, last accessed April 20 2016).

An important question raised by the previous study was whether any mild trauma would augment host resistance to bacterial pathogens. ${ }^{4}$ To address this issue, a model of peripheral injury was needed for comparison. In our previous clinical study, blunt non-head trauma patients were used as controls, but our murine studies used shaminjured mice. ${ }^{4}$ Traumatic injury models have been shown to have drastic effects on immunity. For example, a pulmonary contusion model showed enhanced neutrophil recruitment and expression of inflammatory mediators in response to an intratracheal lipopolysaccharide challenge. ${ }^{5}$ However, unlike our mTBI model, these neutrophils ultimately were deleterious and caused lung injury. Fracture and pseudofracture models replicate a common clinical injury and can have powerful immunosuppressive effects. ${ }^{6,7}$ These disparate findings show the wide spectrum of immune alterations and the necessity for proper controls in studying traumatic sequelae. Prior publications have raised the concern that any mild trauma could alter the host immune response and that the protective effects seen in our mTBI model are not specific to head trauma. Thus, we sought to develop an injury model that induces similar hematologic, physiologic, and inflammatory changes without compromising the lung, our primary organ of interest. A model of blunt tail trauma was studied to test the hypothesis that only TBI will augment pulmonary antibacterial defenses. The current study also showed that specific blockade of substance P (SP) signaling decreased survival to a pulmonary pathogen only in mTBI mice, confirming the mechanism of the improved survival. ${ }^{4}$ The significance of this work is highlighted further by a 2015 report showing that TBI patients had increased levels of SP. ${ }^{8}$ The mechanism of improved survival occurs through enhanced phagocytic cell clearance of bacteria.

\section{Materials and Methods}

\section{Animals}

Female ICR mice (Harlan Sprague Dawley, Indianapolis, IN) were used for all experiments. All mice were 8 to 10 weeks old, weighed between 24 and $30 \mathrm{~g}$, and were acclimated for at least 3 days before experiments. Animals were housed in a temperature- and humidity-controlled facility with a 12-hour light/dark cycle. Food and water were provided ad libitum. The Institutional Animal Care and Use Committee at Boston University approved all experiments. The neurokinin-1 receptor (NK1R) is the preferred receptor for SP. ${ }^{9}$ The NK1R antagonist, aprepitant $(50 \mathrm{mg} / \mathrm{kg}$; Merck, Kenilworth, NJ; 2\% carboxymethylcellulose; Sigma, St. Louis, MO) was given 2 hours before trauma and afterward at 12-hours intervals by oral gavage (p.o.) until pneumonia instillation. Vehicle mice were administered $2 \%$ carboxymethylcellulose p.o.

\section{Trauma and Pneumonia Models}

mTBI was performed as previously described. ${ }^{4}$ Mice were evaluated 2 hours after injury using our Modified Mouse Coma Scale (MMCS) (Table 1) by two blinded evaluators (M.H.V. and B.L.L.) and only mice meeting the criteria for mild TBI (MMCS > 13) were included. Of the mTBI mice, $>95 \%$ had a MMCS $>13$. To induce mild tail trauma (TT), mice were anesthetized using isoflurane and placed in a prone position on the surface of the mTBI apparatus. The $170 \mathrm{~g}$ steel rod was released from a height of $8.5 \mathrm{~cm}$ onto a spot $2.0 \mathrm{~cm}$ from the base of the tail. This height was chosen based on pilot studies in which it induced neutrophilia at 4 hours similar to the mTBI mice. Immediately after impact the mouse was removed and given a subcutaneous injection of $0.05 \mathrm{mg} / \mathrm{kg}$ buprenorphine in $1 \mathrm{~mL}$ of normal saline. The

Table 1 Modified Mouse Coma Scale

\begin{tabular}{|c|c|c|c|c|c|}
\hline \multicolumn{3}{|c|}{ Human Glasgow Coma Scale } & \multicolumn{3}{|c|}{ Modified Mouse Coma Scale } \\
\hline \multirow[t]{3}{*}{ Eye opening } & Spontaneous & 4 & Posture & Moving about cage & 4 \\
\hline & To command & 3 & & Hunched & 3 \\
\hline & None & 1 & & Prone & 1 \\
\hline \multirow[t]{3}{*}{ Verbal response } & Oriented & 5 & Response to sound & Turns to investigate & 5 \\
\hline & Confused, disoriented & 4 & & Moves away & 4 \\
\hline & None & 1 & & No response & 1 \\
\hline \multirow[t]{4}{*}{ Motor response } & Obeys commands & 6 & Tail lift response & Extension and reaching & 6 \\
\hline & Localizes pain & 5 & & Extension without reaching & 5 \\
\hline & Withdraws & 4 & & Lifts head flexion & 4 \\
\hline & Abnormal flexion to pain & 3 & & Flexion no head lift & 3 \\
\hline
\end{tabular}


mTBI mice also received the same dose of buprenorphine. Mice were placed on a warming pad until they were able to right themselves. Mice were selected randomly to undergo TBI, tail, or sham injury. Sham injury mice were anesthetized, placed in the apparatus, but no trauma was induced, although they were given the same dose of buprenorphine as the mTBI mice to keep the groups equivalent. Pneumonia was induced 48 hours after trauma by administration of 0.5 to $1 \times 10^{8}$ colony forming units (CFU) of Pseudomonas aeruginosa (Boston 41501; ATCC, Manassas, VA) in $50 \mu \mathrm{L}$ of Hank's balanced salt solution by intratracheal instillation as previously described. ${ }^{4}$ The majority of the current data do not include a sham-injured group because the study attempts to determine if any trauma will augment survival to a pathogen challenge. Not including the sham-injured group complies with the three $\mathrm{R}$ ethics of use of animals in science (replacement, reduction, and refinement), specifically the reduction component. ${ }^{10}$

\section{Hematologic Analysis}

Blood was collected $(20 \mu \mathrm{L})$ from the facial vein at serial time points into $180 \mu \mathrm{L}$ of phosphate-buffered salineEDTA (50:1) and centrifuged for 5 minutes at $1000 \times g .{ }^{11}$ Plasma later was removed for later analysis while the pellets were resuspended in Hemavet diluent and used to obtain a complete blood count with differential using a Hemavet 950 instrument (Drew Scientific, Miami Lakes, FL).

\section{Physiologic and Respiratory Parameters}

Physiologic measurements (heart rate, $\mathrm{O}_{2}$ saturation, and pulse distension) were obtained by cervical collar telemetry (MouseOx; Starr Lifesciences, Oakmont, PA). Respiratory measurements (respiratory rate, time of inspiration/expiration, and tidal volume) also were measured using unrestrained whole-body plethysmography (Buxco Research Systems, Wilmington, NC). Initial readings were taken on the day before trauma and then at 4 hours, 24 hours, and 48 hours after injury.

\section{Tissue and Plasma Samples}

Mice were sacrificed under ketamine/xylazine anesthesia. Bronchoalveolar lavage (BAL) was performed 4 hours after instillation of Pseudomonas with $5 \mathrm{~mL}$ of warm Hank's balanced salt solution-EDTA $(50: 1)$ in $500-\mu \mathrm{L}$ aliquots. Of the first $1 \mathrm{~mL}, 100 \mu \mathrm{L}$ was taken to determine the bacterial burden by serial dilutions on 5\% sheep's blood agar plates. Cell pellets were combined and counted using a Beckman-Coulter particle counter (Coulter Electronics, Danvers, MA). A cell differential was performed by counting 300 cells spun using a Cytospin (Shandon, Waltham, MA) and slides were stained with Diff-Quick (SIEMENS Healthcare Diagnostics, Newark, DE). Mice were sacrificed at 30 minutes after mTBI to collect blood and BAL fluid. SP concentrations in samples were measured by enzyme-linked immunosorbent assay using a commercial kit (Substance P Enzyme-Linked Immunosorbent Assay Kit; Cayman Chemical Co, Ann Arbor, MI).

\section{Phagocyte Killing of Bacteria}

Neutrophils $\left(2 \times 10^{5}\right)$ in whole blood were obtained via retroorbital exsanguination or neutrophils $\left(5 \times 10^{5}\right)$ were isolated using a PerColl gradient from bone marrow. Hemavet analysis was performed on whole blood for normalization of neutrophil number. Cytospins of bone marrow were performed to ensure efficient separation. Samples were incubated at a multiplicity of infection of 5 with Pseudomonas for 1 hour at $37^{\circ} \mathrm{C}$ in a final volume of $200 \mu \mathrm{L}$. Two control samples of bacteria only were incubated to provide a control. After 1 hour, cells were lysed in $\mathrm{H}_{2} \mathrm{O}$ followed by serial dilutions in Hank's balanced salt solution onto 5\% sheep's blood agar plates. The microbicidal capacity was calculated as the percentage of killing $=\left(\mathrm{CFU}_{\mathrm{bac}}-\mathrm{CFU}_{\text {sample }}\right) /\left(\mathrm{CFU}_{\mathrm{bac}}\right) \times 100$. During the development of the phagocyte killing assay, neutrophils from sham-injured animals were first studied. Because the bacterial killing by sham-injured animals was similar to TT animals the results were pooled to increase the power of the study.

\section{Injury Quantification}

To quantify the extent of plasma leakage from the vasculature, $1 \%$ body weight of a $1 \%$ Evans blue solution was injected i.p. 1 hour before trauma. Mice were sacrificed 48 hours after trauma using ketamine/xylazine anesthesia, then perfused with phosphate-buffered saline-heparin $(10 \mathrm{U} / \mathrm{mL})$ followed by $10 \%$ formalin. Whole brains and a $1.5-\mathrm{cm}$ long section of the tail encompassing the injury site were collected and stored in 10\% formalin. Two-millimeter (brain) or 3-mm (tail) slices were imaged using an Odyssey near-infrared scanner (Li-Cor, Lincoln, NE) to measure Evans' blue extravasation from the vasculature and penetration into the tissue. After Li-Cor imaging, tissue slices then were embedded in paraffin blocks for sectioning, hematoxylin and eosin staining, and histologic analysis. Glass slides were scanned on an iScanCoreo (Ventana, Tucson, AZ).

\section{Statistical Analysis}

Statistical analyses were performed using Prism software version 5.0 (GraphPad, La Jolla, CA). Hematologic, physiological, and pulmonary data were analyzed by two-way analysis of variance, with Bonferroni post-tests where appropriate. SP BAL levels were below the detection limit in naive mice and a Fisher exact test was used to compare the levels with the injured groups. Survival data were analyzed by log-rank tests. Comparisons between mTBI and TT after pneumonia challenge and microbial killing were made using a two-tailed $t$-test. 


\section{Results}

\section{TT and mTBI Induce Similar Inflammatory Hematologic Changes}

To test the hypothesis that only mTBI primes the immune system, we developed a model of peripheral injury with a similar level of injury as our mTBI. TT caused by a weight being dropped on the tail was chosen because it avoids several potential confounders. First, our studies focused on the immune response to pneumonia and TT would not compromise pulmonary or diaphragmatic function.,12 Second, this model closely mimicked our closed-head mTBI model that avoided skin manipulation and potential damage to organs implicated in mounting immune responses. ${ }^{4}$ Third, as in our mTBI model, we avoided bone fractures that are known to be immunosuppressive. ${ }^{6,7}$ Because virtually any trauma induces an inflammatory response, including neutrophilia related to the severity of the injury, we first analyzed hematologic changes. Both the mTBI and TT groups showed similar early neutrophilia 4 hours after injury (Figure 1A), indicating that both models induced a detectable level of trauma. Concomitantly, the lymphocyte concentration in both groups decreased in an expected fashion (Figure 1B). The total white blood cell count did not differ significantly (Figure 1C). There were no significant differences between trauma groups at the time points analyzed by two-way analysis of variance. Comparable increases in neutrophils and decreases in lymphocytes suggest that neither model induced a higher level of trauma. The normalization of hematologic alterations by 48 hours suggests that differences in circulating immune cell numbers does not contribute to enhanced immune responses.

\section{TT and mTBI Induce Physiologic Responses That Normalize within 48 Hours}

Additional experiments sought to characterize changes after injury to determine whether physiologic differences were induced by the trauma and if they persisted up to the time of the pneumonia challenge. Physiological measurements of heart rate, pulse distension (a measure of local arterial blood flow proportional to cardiac output), and $\mathrm{O}_{2}$ saturation were measured using cervical collar telemetry. The telemetry data allowed repeated measurements on the same experimental subject to see the onset and resolution over the 48 hours after injury. There were no significant differences between TT and mTBI mice in heart rate during the 48 hours after injury (Figure 2A). Although pulse distension (Figure 2B) was significantly higher in TT mice, and $\mathrm{O}_{2}$ saturation (Figure 2C) was lower at 4 hours after trauma, these normalized by 24 hours.

Because we would be inducing a pulmonary infection, additional lung studies were performed with an alternative technology. Respiratory parameters were measured by unrestrained whole-body plethysmography. ${ }^{13}$ Tidal volume remained essentially unchanged in both trauma models (Figure 3A). Both trauma models had a significant decrease in respiratory rate (Figure 3B) as a result of increased times of inspiration (Figure 3C) and expiration (Figure 3D), with greater changes in the mTBI group. These results show that the relatively minor, early physiologic, and respiratory differences between models at 4 hours resolved by 24 hours. The systemic measurements support that both models showed similar although not identical responses through the first 48 hours after trauma.

\section{SP Levels in BAL and Plasma}

Pilot studies have shown that both BAL and plasma levels of SP peaked within 30 minutes after either TT or mTBI (data not shown). A group of naive mice were studied to determine systemic as well as local levels of SP. SP was not detectable in the BAL of naive mice, but did increase after either TT or mTBI (Figure 4). Similarly, the plasma SP level was increased in both trauma groups, and the naive mice had low levels as previously reported (ie, $<20 \mathrm{pg} / \mathrm{mL}$ ). ${ }^{14,15}$ These data indicate that either mTBI or TT was sufficient to induce the release of SP into both the BAL and plasma. Figures 1, 2, 3, and 4 show that both trauma models produce a similar initial inflammatory response.
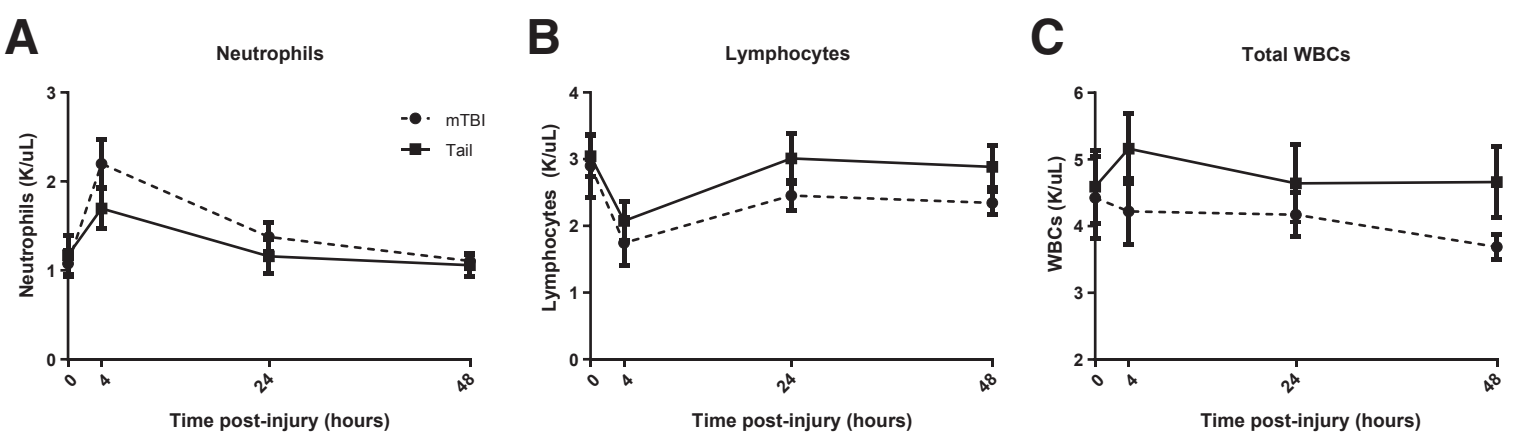

Figure 1 Hematologic changes after brain or tail trauma. Both groups show similar hematologic changes after trauma. A: Trauma induced neutrophilia in both groups, which resolved by 48 hours. B: Lymphocytes decreased at the 4-hour time point in both groups. C: The total white blood count (WBC) was unchanged. Data are shown as means \pm SEM. No significant differences were found by two-way analysis of variance. $n=8$ for mTBI; $n=12$ for tail. 

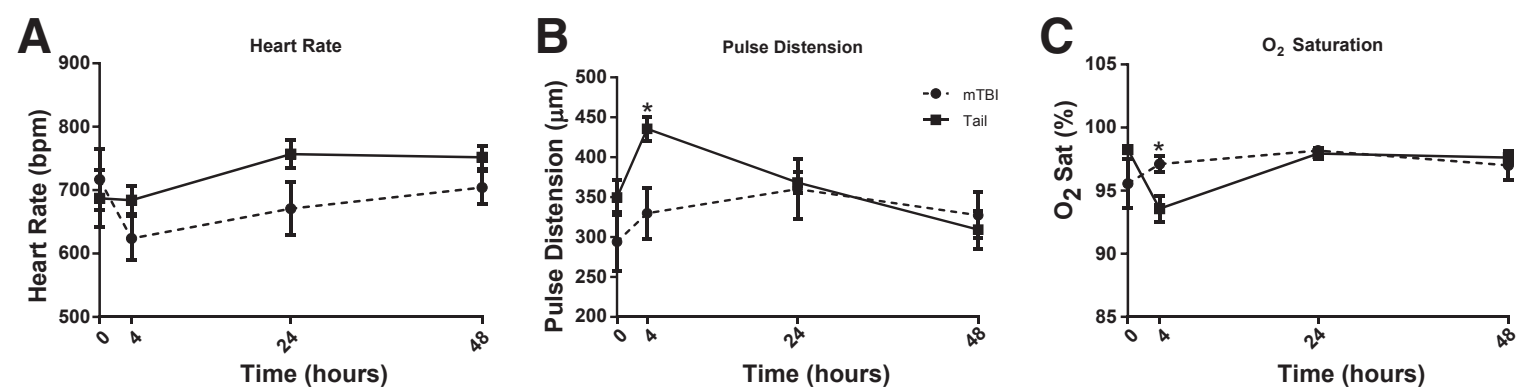

Figure 2 Early hemodynamic changes after trauma. A: There was no significant difference in the heart rate at any time point. Pulse distension was higher in tail trauma (TT) mice at 4 hours (B) and $\mathrm{O}_{2}$ saturation was lower (C), however, both returned to normal within 24 hours. Hemodynamic data were collected from cervical collar telemetry (Mouse0x; Starr Lifesciences). Data are shown as means \pm SEM. $n=5$ for $\mathrm{mTBI} ; n=8$ for tail. ${ }^{*} P<0.05$ mild traumatic brain injury (mTBI) versus tail at the indicated time point.

\section{Assessing Neurologic Injury: MMCS}

Our data indicate similar systemic responses to trauma but additional experiments were performed to quantify the severity of neurologic injury in our mice. The Glasgow Coma Scale is the most commonly used neurologic injury scale for adults for traumatic head injuries. ${ }^{16}$ We adapted the Glasgow Coma Scale criteria to mice (MMCS) by observing their spontaneous behavior, response to auditory stimuli, and motor reflex responses similar to a clinical evaluation. The MMCS allowed us to assess the severity of neurologic deficits quickly and accurately (Table 1), as well as limit interobserver variability. To mimic the clinical scenario of a patient presenting to the emergency department for evaluation after head injury, two blinded investigators (M.H.V. and
B.L.L.) first scored the mice 2 hours after trauma. This time point was chosen to recapitulate the common delay between injury and clinical evaluation, as well as the superior performance of the 2-hour evaluation as a predictor of outcome. ${ }^{17}$ These same mice were also evaluated at 24 and 48 hours after trauma to longitudinally track any deficits in neurologic function. The same Glasgow Coma Scale score for TBI severity was used in our MMCS, as follows: mild was indicated by a score of 13 to 15 , moderate was indicated by a score of 9 to 13 , and severe was indicated by a score of $<8$. Our mTBI model reproducibly generated scores in the mild range and TT mice showed no deficits at all (Figure 5). The results show that our TBI model consistently was in the mild category and the MMCS can be used as a robust tool for assessing head trauma severity in mice.
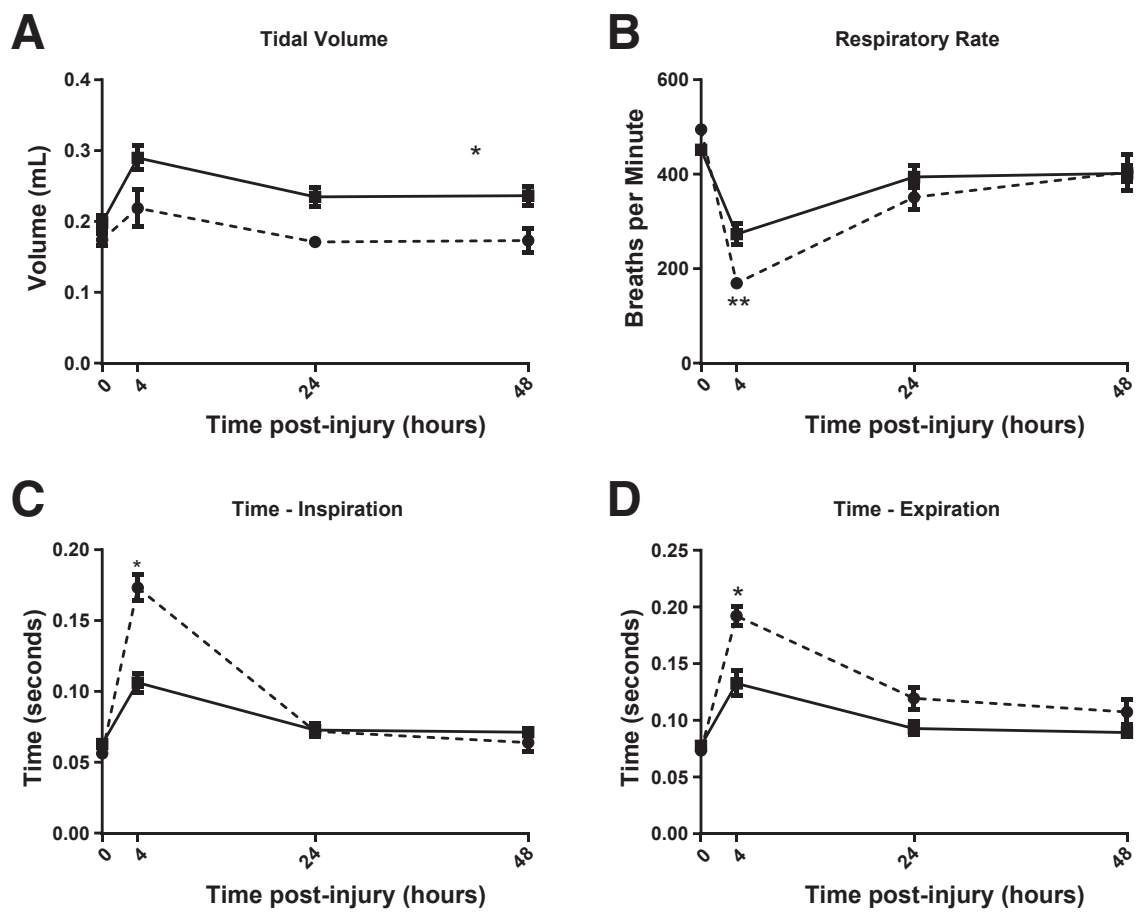

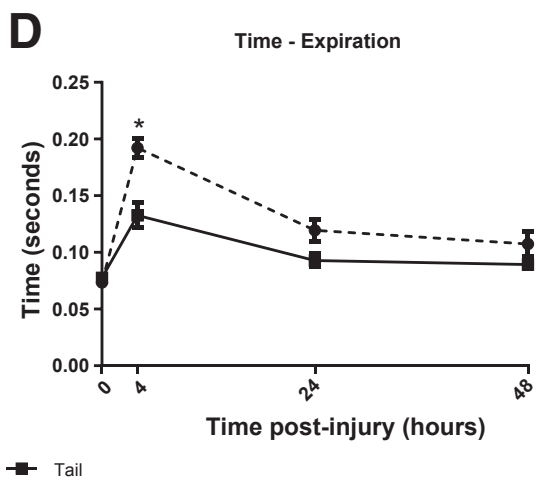

Figure 3 Pulmonary physiology measured by whole-body plethysmography. Both groups of trauma show similar respiratory parameters by 48 hours after trauma. A: Mild traumatic brain injury (mTBI) mice had lower tidal volumes overall but showed the same trend as tail trauma while returning to their baseline level by 24 hours. Respiratory rate (B), time of inspiration (C), and time of expiration (D), were significantly different between groups only at the 4-hour time point, but normalized by 24 hours. Data were obtained by unrestrained whole-body plethysmography at the time points indicated. Data are shown as means \pm SEM. $n=5$ for mTBI; $n=8$ for tail. ${ }^{*} P<0.001,{ }^{*} P<<0.01$ tail trauma compared with mTBI. 

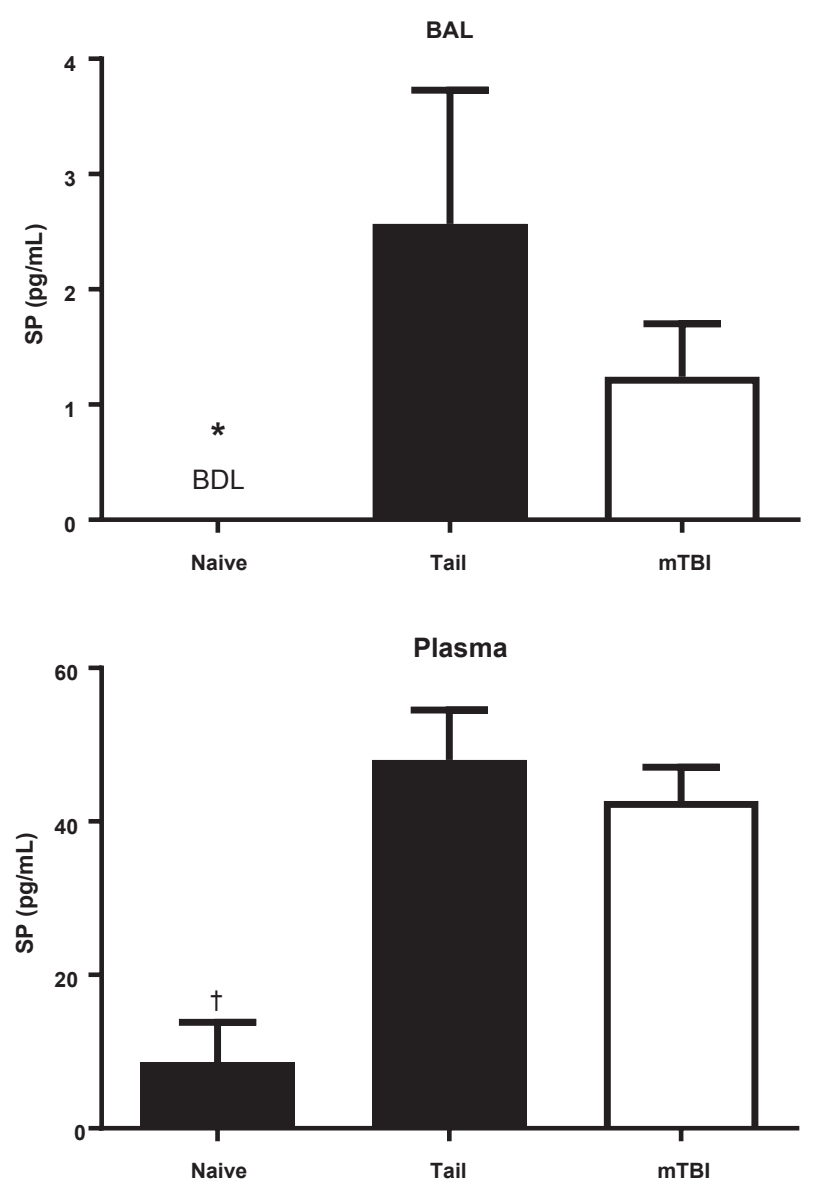

Figure 4 Substance $P(S P)$ levels in naive mice or 30 minutes after tail trauma or mTBI. Mice were subjected to tail trauma or mild traumatic brain injury (mTBI) and sacrificed 30 minutes later, naive mice had no manipulation. SP was increased significantly in the BAL and plasma of both injured mice. There was no difference in the concentrations of SP between the injured groups. $n=4$ for naive mice; $n=8$ for tail and mTBI. ${ }^{*} P<0.05$ versus the injured groups by the Fisher exact test for bronchoalveolar lavage (BAL); ${ }^{\dagger} P<0.05$ by analysis of variance with the Tukey correction compared with the injured groups for plasma. BDL, below detection limit $(0.1 \mathrm{pg} / \mathrm{mL})$.

\section{Characterization of Injury Sites}

To characterize the location of injury after trauma, we performed detailed analyses of tail and brain tissue from both groups at 48 hours after injury (ie, immediately before pulmonary pathogen challenge). Evans' blue dye was injected intraperitoneally before the trauma to allow us to visualize and quantify the injury. Evans' blue is a fluorescent dye that binds to serum albumin with high affinity and is unable to cross the intact blood brain barrier. These properties allowed us to identify the area of injury, notably extravasation from the vasculature into tissue spaces, by its blue coloration, as well as quantify the signal intensity using the Li-Cor Odyssey near-infrared scanner.

\section{Near-Infrared Quantification}

Evans' blue fluorescence can be detected at $700 \mathrm{~nm}$ using the Odyssey near-infrared scanner. mTBI mice showed an

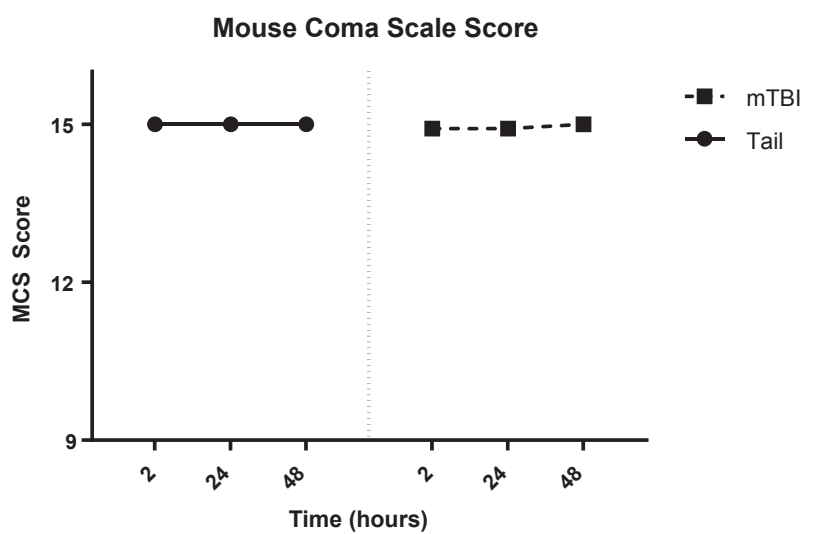

Figure 5 Modified Mouse Coma Scale (MMCS) scores. The MMCS showed no significant neurologic impairment in the mild traumatic brain injury (mTBI) model. Animals were evaluated by two blinded investigators (M.H.V. and B.L.L.) at each time point using our MMCS (Table 1). Tail trauma mice had no deficits in scoring at any time points. No significant differences were found between groups at all time points. Data are shown as means \pm SEM. $n=6$ per group.

intense signal in the area of injury that reflected the histologic damage described later (Figure 6A). TT brains had a slight signal owing to the blood vessels in the brain (Figure 6A). Accordingly, TT tails reflect intense signal throughout the sections whereas mTBI tail signal was limited to the vasculature (Figure 6D).

\section{Hematoxylin and Eosin Sections}

Brains of mTBI mice showed focal lesions with acute necrosis and hemorrhage generally involving the anterior and ventral portions of the brain. The ventral pallidum and anterior commissure were the most common structures damaged in the brains. No lesions were identified in any of the brains of TT mice (Figure 6B). Tails of TT mice reflect the damage seen grossly with areas of extravasated red blood cells in the tissue space, and infiltration of neutrophils (Figure 6E). None of these changes were observed in the tails of the MTBI mice.

For both tissues, the positive dye signal appears to be larger than the extravasation seen microscopically, probably owing to the increased sensitivity to damage using Evans' blue dye compared with hematoxylin and eosin-stained sections. ${ }^{18}$ Quantification of the dye intensity shows that the injury was isolated to the targeted tissue (Figure 6, C and F). The combination of the near-infrared quantification and histologic observations indicated the localization of trauma between these models.

\section{mTBI Specifically Enhances Resistance to Pneumonia}

Because both trauma groups induced similar physiologic and hematologic responses that normalized by 24 to 48 hours, we proceeded to study whether only mTBI induced pulmonary immune priming to help eradicate pathogens. Each trauma group was challenged 48 hours after injury with 0.5 to $1.0 \times 10^{8} \mathrm{CFU}$ of Pseudomonas intratracheally using a well-described nonsurgical model. ${ }^{19,20}$ mTBI mice 
A
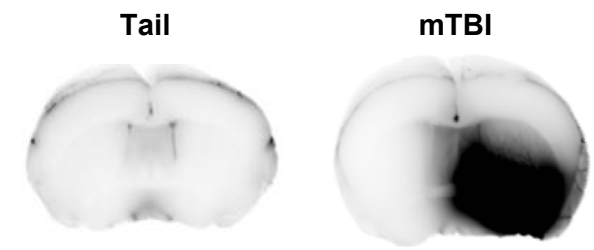

D

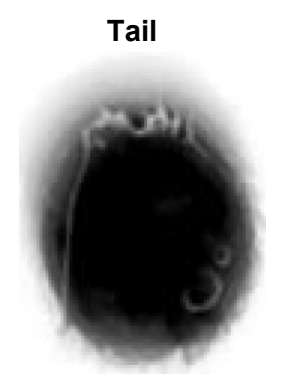

B
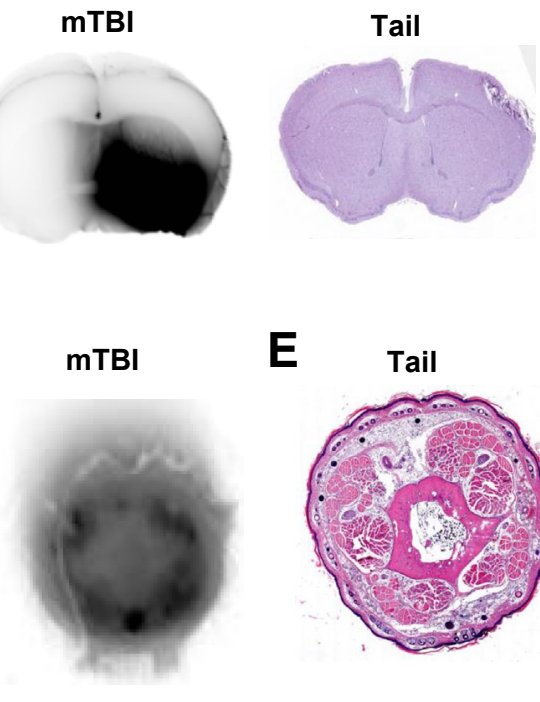

$\mathbf{E}$

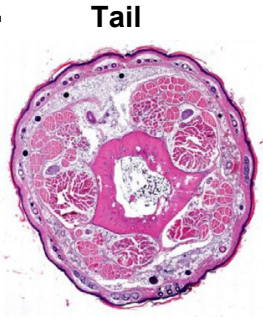

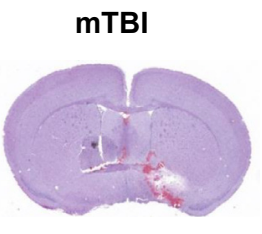

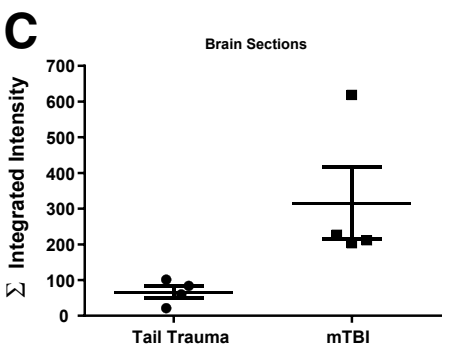

$\mathbf{F}$
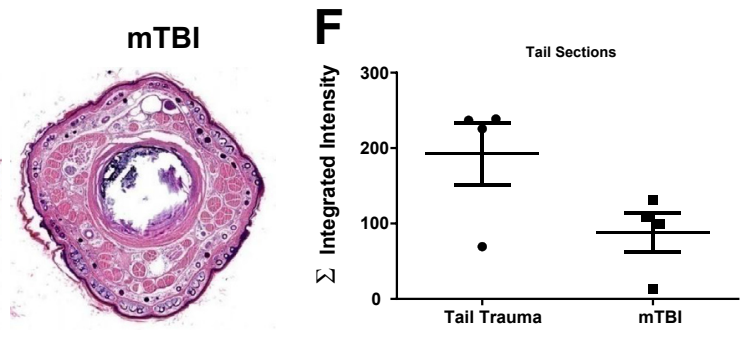

Figure 6 Evaluation of trauma localization. Trauma is limited to the injured region by histologic analysis and Evans' blue quantification. A: Near-infrared imaging of fixed brain slices shows an intense signal in mild traumatic brain injury (mTBI) (right) but not tail trauma mice (left), where it was limited to the vasculature. B: Hematoxylin and eosin staining confirms localization of the damage. C: Quantification of albumin extravasation by near-infrared scans shows that mTBI mice have higher signals across the measured sections. D: Near-infrared imaging indicates the presence of Evans' blue leakage of tail trauma (TT) mice (left) whereas mTBI mice (right) have intensities limited to vascular regions. E: Hematoxylin and eosin sections confirm localization of damage in the tail. F: Quantification of Evans' blue fluorescence shows increased signal in the tails of TT mice. Data are shown as means \pm SEM $(\mathbf{C}$ and $\mathbf{F})$. $n=4$ per group.

showed significantly improved 7-day survival compared with TT mice (76.5\% versus $40 \%$ ) (Figure $7 \mathrm{~A})$. In fact, TT mice survived at a rate similar to sham mice, and thus in future experiments the sham group was combined or omitted following Animal Research: Reporting of in Vivo Experiments guidelines to reduce the number of mice used. ${ }^{10}$ TT or mTBI mice were challenged with Pseudomonas 48 hours after trauma and BAL was performed 4 hours after infection. An enhanced pulmonary response to the pathogen was mTBI specific with fourfold increased neutrophil recruitment to the airspaces compared with TT (Figure 7B). mTBI mice also have significantly reduced bacterial numbers in the alveolar spaces (Figure 7C). Early neutrophil recruitment has been shown previously to be a key determinant of outcomes in serious infections. ${ }^{11}$ This improved recruitment and diminished bacterial burden found in mTBI mice is reflected in their improved survival. The enhanced neutrophil recruitment and bacterial elimination warranted further studies to elucidate the specific mechanism(s) by which mTBI reduced bacterial burden.

\section{Phagocytic Cells from mTBI Mice Show Enhanced Killing of Bacteria}

These findings confirm our previous results that mTBI enhances pulmonary defenses against bacterial pneumonia. ${ }^{4}$ However, whether increased pathogen clearance was solely the result of the increased polymorphonuclear nucleocyte influx or enhanced microbicidal capacity is unknown. To determine this, we mixed phagocytes from peripheral blood
A

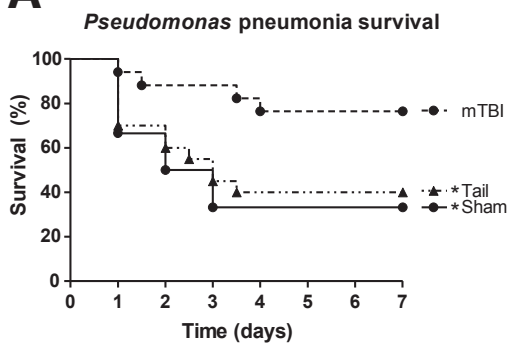

B

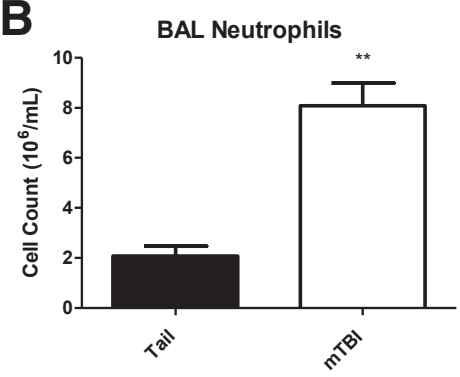

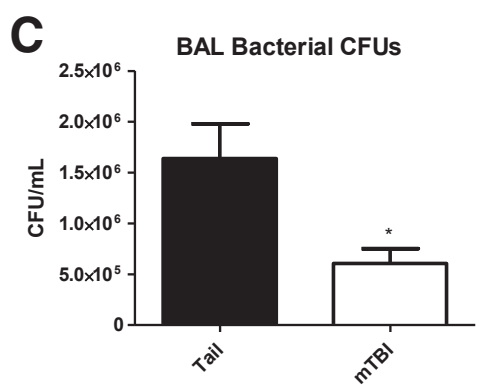

Figure 7 Response to pulmonary pathogen challenge after tail trauma or mild traumatic brain injury (mTBI). A: mTBI mice survive at higher rates than either sham injury or tail trauma mice to a Pseudomonas pneumonia challenge $(76.5 \%$ versus $33 \% / 40 \%, n=17,6$, and 20 , respectively). B: At 4 hours after pneumonia, mTBI mice recruit significantly more neutrophils to the airspace compared with tail trauma mice as determined by the number recovered in the bronchoalveolar lavage (BAL). C: mTBI mice have significantly reduced bacterial burdens in the BAL fluid. Data are shown as means \pm SEM. $n=11 \mathrm{mTBI}$; $n=12$ tail. ${ }^{*} P<0.05,{ }^{* *} P<0.001$. CFU, colony forming units. 


\section{Peripheral Blood}
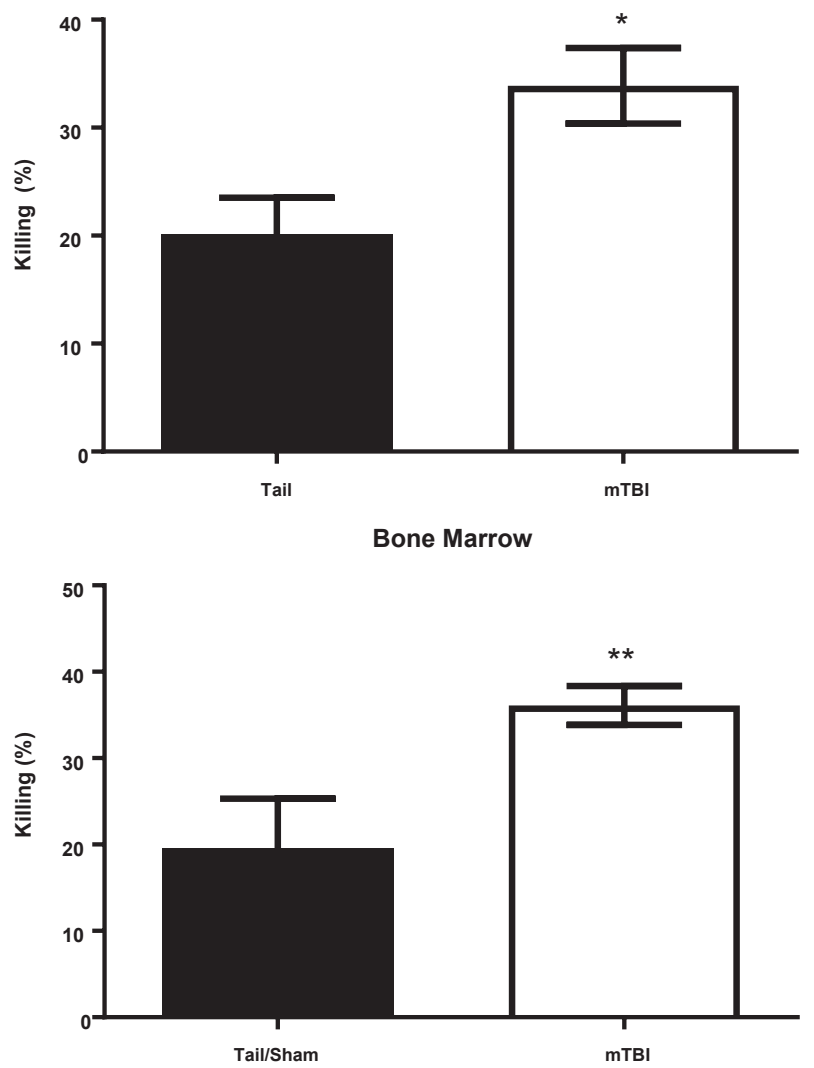

Figure 8 Comparison of Pseudomonas killing by phagocytes. Phagocytes from both peripheral blood and bone marrow of mild traumatic brain injury (mTBI) mice show increased microbicidal activity compared with tail/sham mice. Polymorphonuclear nucleocytes were incubated for 1 hour with Pseudomonas at a multiplicity of infection of 5 , then diluted serially to calculate the percentage of killing compared with no cell control. The percentage of killing was calculated as described in Materials and Methods, in which an increased percentage of killing indicates better polymorphonuclear leukocyte function. Data are shown as means \pm SEM. Blood: $n=9$ (tail); $n=10$ (mTBI). Marrow: $n=17$ (tail); $n=21$ (mTBI). ${ }^{*} P<0.05,{ }^{*} P<<0.01$.

or bone marrow with Pseudomonas and quantified the ability to kill bacteria (with higher numbers indicating enhanced pathogen clearance). Cells from mTBI mice had a significantly increased ability to kill bacteria compared with TT mice (Figure 8). These data support that mTBI enhances resistance to pneumonia through two synergistic mechanisms: increased recruitment of neutrophils and enhanced microbicidal function.

\section{SP Signaling Is Crucial to the Enhanced Resistance to Pneumonia}

SP signaling is known to improve neutrophil chemotaxis, cytokine production, and reactive oxygen species generation. ${ }^{21-23}$ Our previous work showed the critical role of SP in mTBI priming of the host response in which antagonism of the primary SP receptor, NK-1R, abrogated the mTBI survival benefit. ${ }^{4}$ Our hypothesis is that mTBI causes rapid release of SP to increase the host response to a pulmonary pathogen. However, both TT and MTBI caused a similar increase in peak levels of SP, therefore further studies are needed to determine if an SP antagonist also would alter survival in TT mice.

The SP antagonist used in the original manuscript ${ }^{4}$ is no longer available. However a different NK-1R antagonist, aprepitant, is an Food and Drug Administration-approved drug for chemotherapy-induced nausea/vomiting. Because SP was induced so rapidly, aprepitant was administered by oral gavage 2 hours before trauma and every 12 hours until 1 hour before Pseudomonas instillation. Administration before trauma and through the period after trauma was chosen to negate potential effects of SP release after the injury. NK-1R blockade with aprepitant eliminated the survival benefit of mTBI (Figure 9), supporting our previous findings. ${ }^{4}$ Aprepitant treatment did not alter survival of TT mice. mTBI plus vehicle mice had significantly less mortality than the other groups. These studies confirm that SP signaling is a specific and critical mechanism in the enhanced host response found in our mTBI model.

\section{Discussion}

Although TBIs are a prevalent problem with approximately 2.5 million TBI-related emergency department visits, hospitalizations, or deaths in the United States each year (CDC, http://www.cdc.gov/traumaticbraininjury/get_the_facts.html,

\section{Pseudomonas pneumonia survival}

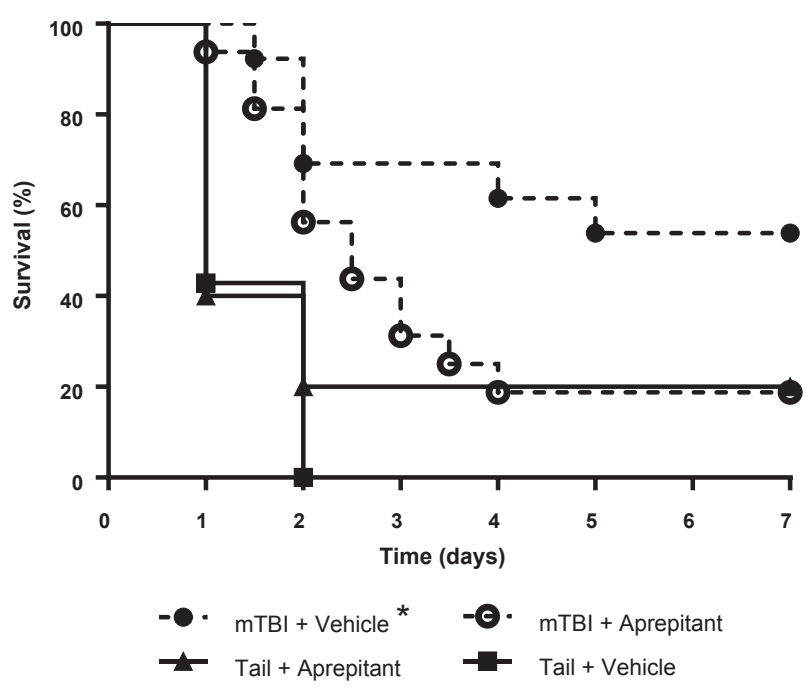

Figure 9 Substance $P$ modulation of traumatic brain injury (TBI) + Pseudomonas mortality. Blockade of substance P signaling abrogates the survival benefit only in mild TBI (mTBI) mice. Aprepitant was administered 2 hours before trauma, and every 12 hours up until 1 hour before pneumonia. Mice were monitored for 14 days, no deaths occurred after 7 days. Survival curves were analyzed by log-rank (Mantel-Cox) test. $n=6$ (tail + vehicle); $n=5$ (tail + aprepitant) $n=13$ (mTBI + vehicle); $n=16$ (mTBI + aprepitant). ${ }^{*} P<0.05$ mTBI + vehicle versus mTBI + aprepitant. 
last accessed April 20, 2016), this study investigated the poorly understood protective mechanism after mTBI. Our focus was on mTBIs because these patients do not have immediate neurologic sequelae, but actually show enhanced resistance to pneumonia. ${ }^{4}$ Our mTBI model was designed to investigate insights into the neuroimmune axis to develop new host-driven therapies to fight pathogens. Interestingly, our previous work showed that mTBI enhances the resistance to pneumonia compared with sham-injured mice. ${ }^{4}$ However, valid concerns were expressed whether any trauma would produce a similar protective effect. The goal of this study was to generate a suitable control trauma model to answer whether immune priming is specific to $\mathrm{mTBI}$. We propose that our TT model accomplishes this goal by inducing similar early responses after trauma. These results show that only mTBI increases bacterial clearance, neutrophil recruitment, and survival compared with TT. This TT model will serve as an appropriate control for future studies investigating the mechanisms of immune augmentation. Furthermore, the use of this model is clinically relevant because studies would compare TBI patients with blunt, non-head trauma patients of similar injury severity instead of healthy subjects. By accounting for potential confounding variables in preclinical models, we hope to improve translation of our research to patients. Thus, having a proper control model is important to determine specific mechanisms by which our mTBI model can enhance immune responses.

Although immune modulation is not a novel idea, major discoveries in dampening its response have garnered most of the attention. Enhancing its function is now coming to the forefront as a potential method to combat antibiotic resistance because resistant organisms are becoming an increasingly difficult problem to overcome. These pathogens are estimated to cause 23,000 deaths in the United States and $\$ 20$ billion in excess health care costs per year (CDC, http://www.cdc.gov/drugresistance/threat-report2013, last accessed April 20, 2016). Recently, the US government doubled its budget for combating antibiotic resistance to $\$ 1.2$ billion to combat this epidemic. The explosion of antibiotic-resistant organisms coupled with the slow generation of effective new antibiotics requires the development of new strategies to combat these pathogens. Our current pharmacologic arsenal may be ineffective against resistant pathogens, but prior evidence has shown the pathogens still may be susceptible to host innate immune defenses. $^{24,25}$ Pathogens themselves may alter innate immune responses as a result of modifications acquired alongside resistance. ${ }^{26}$ Host defenses, especially neutrophils, play an important role as the frontline of containment and resolution of infections. Thus, augmenting innate immune responses may provide an effective alternative to antibiotics. Our mTBI model, and TT control, will allow further investigation into pathways to be exploited for neuroimmune modulation.

The similarity of SP measurements at 30 minutes in both trauma groups appears to conflict with later evidence that only mTBI shows enhanced immune responses blocked by SP-receptor antagonism. There are several possible explanations. It is possible that mTBI induces longer-duration SP signaling through central stimulation of the vagus nerve. Traumatic injuries resolve through down-regulation of inflammation and up-regulation of tissue repair processes. In the case of mTBI, that inflammatory nidus could continue vagal stimulation to prolong the release of SP that was not captured by our methods. The peripheral stimulation by TT probably does not release SP at the same level or duration after injury. Peak levels of SP may be similar at the time points measured, but the total area under the curve may be significantly different, which results in priming of the immune system specific to mTBI. It also is possible that mTBI will result in different degradation products of SP produced by endopeptidases, resulting in better activation of neutrophils. ${ }^{27}$ Finally, there may be differential regulation of the neurokinin receptor in mTBI compared with TT, including increased expression of the truncated NK1R. ${ }^{28}$ These will need to be explored in further studies.

These results confirm our previous work that SP is a critical mediator of immune priming after mTBI using a different NK1R inhibitor. ${ }^{4}$ Aprepitant, a Food and Drug Administration-approved NK1R antagonist for chemotherapy-induced nausea/vomiting, similarly eliminated the enhanced resistance against pneumonia found in mTBI mice. Although effective against chemotherapyinduced nausea/vomiting, aprepitant treatment potentially could endanger patients by further blunting their already limited immune responses, as suggested in previous clinical trials. ${ }^{29}$ We hypothesize that mTBI initiates vagal signaling to release SP that leads to primed host responses to microbial infections. SP signaling has been shown previously to have numerous positive effects on immune cells, such as increased chemotaxis, phagocytosis, and reactive oxygen species production. ${ }^{21-23}$ Further investigation is necessary to determine the precise role of SP in enhancing innate immunity in our model. Despite the physiologic similarities between the models before infection, only mTBI provides protection against an infectious challenge. Thus, our TT model will serve as an appropriate mild trauma control for future studies into the mechanism of neuroimmune priming found in our mTBI model.

These results show that TT serves as an excellent mild trauma control. Only mTBI specifically augments the innate immune response to pneumonia, TT does not. This model allows us to study the specific mechanism(s) of mTBI augmenting the innate immune response. We anticipate that immunologic insights elucidated from these models will allow for the development of new host-driven therapies to combat infections.

\section{References}

1. Stein M, Keller SE, Schleifer SJ: Stress and immunomodulation: the role of depression and neuroendocrine function. J Immunol 1985, 135 : $827 \mathrm{~s}-833 \mathrm{~s}$ 
2. Padgett DA, Glaser R: How stress influences the immune response. Trends Immunol 2003, 24:444-448

3. Hazeldine J, Lord JM, Belli A: Traumatic brain injury and peripheral immune suppression: primer and prospectus. Front Neurol 2015, 6:235

4. Yang S, Stepien D, Hanseman D, Robinson B, Goodman MD, Pritts TA, Caldwell CC, Remick DG, Lentsch AB: Substance P mediates reduced pneumonia rates after traumatic brain injury. Crit Care Med 2014, 42:2092-2100

5. Hoth JJ, Wells JD, Hiltbold EM, McCall CE, Yoza BK: Mechanism of neutrophil recruitment to the lung after pulmonary contusion. Shock 2011, 35:604-609

6. Li H, Itagaki K, Sandler N, Gallo D, Galenkamp A, Kaczmarek E, Livingston DH, Zeng Y, Lee YT, Tang IT, Isal B, Otterbein L, Hauser CJ: Mitochondrial damage-associated molecular patterns from fractures suppress pulmonary immune responses via formyl peptide receptors 1 and 2. J Trauma Acute Care Surg 2015, 78:272-279; discussion 279-281

7. Napolitano LM, Koruda MJ, Meyer AA, Baker CC: The impact of femur fracture with associated soft tissue injury on immune function and intestinal permeability. Shock 1996, 5:202-207

8. Lorente L, Martin MM, Almeida T, Hernandez M, Ramos L, Argueso M, Caceres JJ, Sole-Violan J, Jimenez A: Serum substance P levels are associated with severity and mortality in patients with severe traumatic brain injury. Crit Care 2015, 19:192

9. Douglas SD, Leeman SE: Neurokinin-1 receptor: functional significance in the immune system in reference to selected infections and inflammation. Ann N Y Acad Sci 2011, 1217:83-95

10. Fenwick N, Griffin G, Gauthier C: The welfare of animals used in science: how the "Three Rs" ethic guides improvements. Can Vet J 2009, 50:523-530

11. Craciun FL, Schuller ER, Remick DG: Early enhanced local neutrophil recruitment in peritonitis-induced sepsis improves bacterial clearance and survival. J Immunol 2010, 185:6930-6938

12. Nemzek-Hamlin JA, Hwang H, Hampel JA, Yu B, Raghavendran K: Development of a murine model of blunt hepatic trauma. Comp Med 2014, 63:398-408

13. Vaickus LJ, Bouchard J, Kim J, Natarajan S, Remick DG: Assessing pulmonary pathology by detailed examination of respiratory function. Am J Pathol 2010, 177:1861-1869

14. Wang $\mathrm{Y}$, Wang DH: Role of substance $\mathrm{P}$ in renal injury during DOCAsalt hypertension. Endocrinology 2012, 153:5972-5979

15. Amadesi S, Reni C, Katare R, Meloni M, Oikawa A, Beltrami AP, Avolio E, Cesselli D, Fortunato O, Spinetti G, Ascione R, Cangiano E, Valgimigli M, Hunt SP, Emanueli C, Madeddu P: Role for substance p-based nociceptive signaling in progenitor cell activation and angiogenesis during ischemia in mice and in human subjects. Circulation 2012, 125:1774-1786, S1-S19

16. Teasdale G, Maas A, Lecky F, Manley G, Stocchetti N, Murray G: The Glasgow Coma Scale at 40 years: standing the test of time. Lancet Neurol 2014, 13:844-854
17. Lesko MM, Jenks T, O'Brien SJ, Childs C, Bouamra O, Woodford M, Lecky F: Comparing model performance for survival prediction using total Glasgow Coma Scale and its components in traumatic brain injury. J Neurotrauma 2013, 30:17-22

18. Hamer PW, McGeachie JM, Davies MJ, Grounds MD: Evans Blue Dye as an in vivo marker of myofibre damage: optimising parameters for detecting initial myofibre membrane permeability. J Anat 2002, 200:69-79

19. Kim J, Merry AC, Nemzek JA, Bolgos GL, Siddiqui J, Remick DG: Eotaxin represents the principal eosinophil chemoattractant in a novel murine asthma model induced by house dust containing cockroach allergens. J Immunol 2001, 167:2808-2815

20. Bouchard JC, Kim J, Beal DR, Vaickus LJ, Craciun FL, Remick DG: Acute oral ethanol exposure triggers asthma in cockroach allergensensitized mice. Am J Pathol 2012, 181:845-857

21. Sun J, Ramnath RD, Bhatia M: Neuropeptide substance P upregulates chemokine and chemokine receptor expression in primary mouse neutrophils. Am J Physiol Cell Physiol 2007, 293:C696-C704

22. Bar-Shavit Z, Goldman R, Stabinsky Y, Gottlieb P, Fridkin M, Teichberg VI, Blumberg S: Enhancement of phagocytosis - a newly found activity of substance $\mathrm{P}$ residing in its $\mathrm{N}$-terminal tetrapeptide sequence. Biochem Biophys Res Commun 1980, 94: $1445-1451$

23. Sterner-Kock A, Braun RK, van der Vliet A, Schrenzel MD, McDonald RJ, Kabbur MB, Vulliet PR, Hyde DM: Substance P primes the formation of hydrogen peroxide and nitric oxide in human neutrophils. J Leukoc Biol 1999, 65:834-840

24. Mekontso-Dessap A, Honore S, Kirsch M, Plonquet A, Fernandez E, Touqui L, Farcet JP, Soussy CJ, Loisance D, Delclaux C: Blood neutrophil bactericidal activity against methicillin-resistant and methicillin-sensitive Staphylococcus aureus during cardiac surgery. Shock 2005, 24:109-113

25. Deptula A, Gospodarek E: Reduced expression of virulence factors in multidrug-resistant Pseudomonas aeruginosa strains. Arch Microbiol 2010, 192:79-84

26. Ubagai T, Nakano R, Nakano A, Kamoshida G, Ono Y: Gene expression analysis in human polymorphonuclear leukocytes stimulated by LPSs from nosocomial opportunistic pathogens. Innate Immun 2015, 21:802-812

27. Serra MC, Bazzoni F, Della Bianca V, Greskowiak M, Rossi F: Activation of human neutrophils by substance P. Effect on oxidative metabolism, exocytosis, cytosolic $\mathrm{Ca} 2+$ concentration and inositol phosphate formation. J Immunol 1988, 141:2118-2124

28. Tuluc F, Lai JP, Kilpatrick LE, Evans DL, Douglas SD: Neurokinin 1 receptor isoforms and the control of innate immunity. Trends Immunol 2009, 30:271-276

29. dos Santos LV, Souza FH, Brunetto AT, Sasse AD, da Silveira Nogueira Lima JP: Neurokinin-1 receptor antagonists for chemotherapy-induced nausea and vomiting: a systematic review. J Natl Cancer Inst 2012, 104:1280-1292 